\title{
Amplitude Variability of Delta Scuti Stars: 4 CVn
}

\author{
Michel Breger \\ Institut für Astronomie, Universität Wien, Türkenschanzstr. 17, \\ A-1180, Wien, Austria
}

\begin{abstract}
The amplitude variability of $\delta$ Scuti stars is demonstrated by the behavior of the evolved $\delta$ Scuti star $4 \mathrm{CVn}$. The data on $4 \mathrm{CVn}$ covering over $30 \mathrm{yr}$ shows the amplitudes of almost all modes to be extremely variable with a time scales of ten years or longer. The most rapid decrease of amplitude in $V$ is shown by the $7.38 \mathrm{~d}^{-1}$ mode of 4 CVn, which collapsed from $15 \mathrm{mmag}$ in 1974 to $4 \mathrm{mmag}$ in 1976 , and 1 mmag in 1977. Between 1974-6 and $1977-8$ phase jumps of almost half a cycle occurred. While a simple beating model of two close frequencies can be ruled out from the photometric data, the change is compatible with the growth of a new mode between 1976 and 1977 .
\end{abstract}

\section{Introduction}

Many nonradially pulsating $\delta$ Scuti variables show amplitude variability, especially on long time scales. It is possible that this is actually a universal property of all nonradially pulsating $\delta$ Scuti stars, which becomes evident only after observational data covering many years becomes available (e.g. $4 \mathrm{CVn}$, Breger, McNamara, \& Kerschbaum 1990; X Caeli, Mantegazza \& Poretti 1996). The physical reason for this amplitude Variability is presently not understood. The situation is made more difficult by the fact that only few nonradially pulsating $\delta$ Scuti stars have extensive data available.

\section{Amplitude Variability in the Star $4 \mathrm{CVn}$}

The star $4 \mathrm{CVn}$ is probably the best-studied $\delta$ Scuti star with a high degree of amplitude variability with data going back to 1966 . New data from the extensive multisite campaign of the Delta Scuti Network in 1996 (Breger et al. 1999) and the long single-site campaign of 1997 (Breger \& Hiesberger 1999) provide an excellent opportunity to examine the amplitude variability in more detail.

The campaigns detected 34 frequencies of pulsation for $4 \mathrm{CVn}$, with the majority found independently in both studies. Most of the few differences can be ascribed to the fact that the 1997 study was single-site, had a lower frequency resolution, and that $4 \mathrm{CVn}$ possesses amplitude variability.

The difficulty of applying the new multifrequency solution to the older datasets is caused by the fact that the older datasets are much shorter than the recent investigations. A statistical analysis assuming the presence of all 34 frequencies to determine the annual amplitudes can fail, because this method 


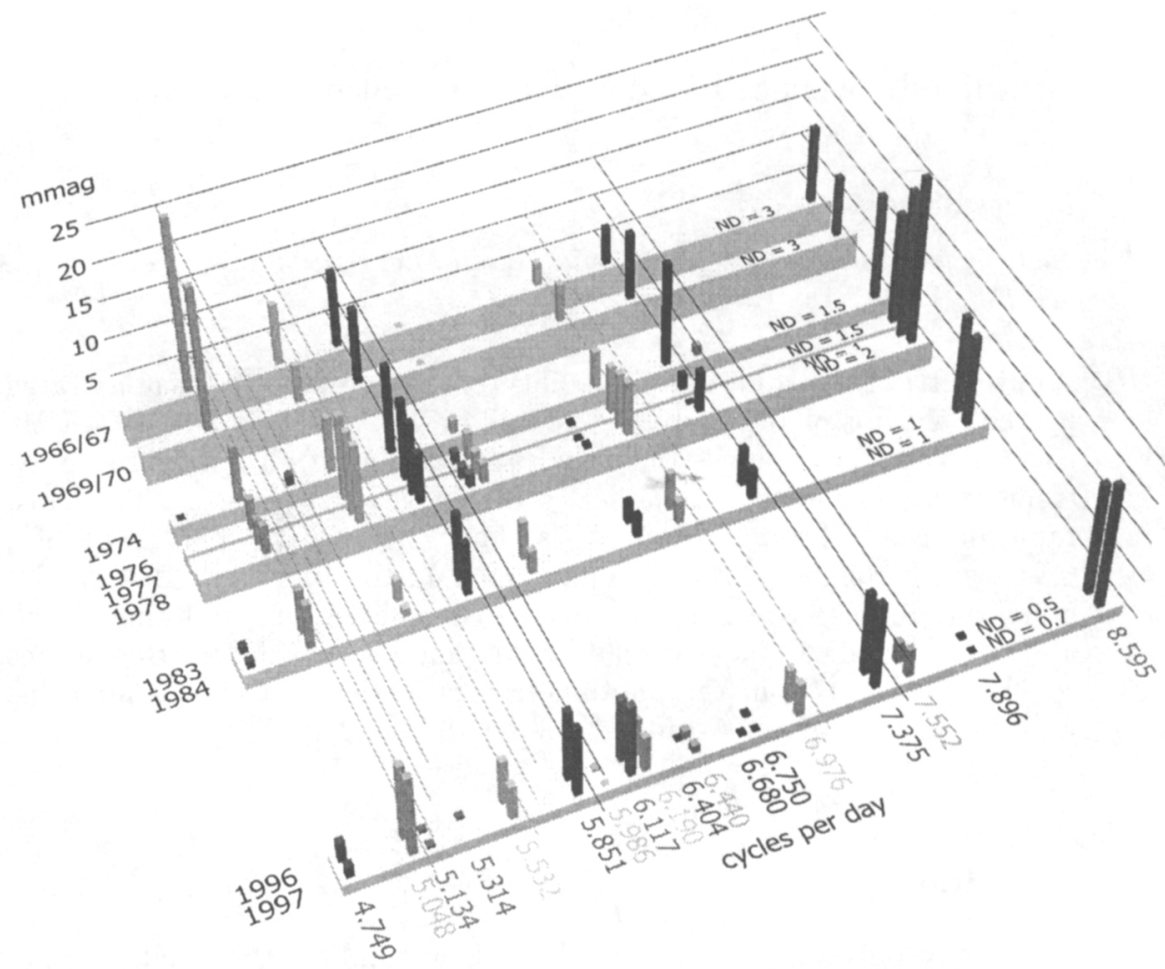

Figure 1. Amplitude variability of $4 \mathrm{CVn}$ from 1966 to 1997 . The amplitude is given by the height of the peaks. The different thresholds for the different years are shown and marked with 'ND'. Amplitudes smaller than these limits are not shown.

requires the determination of $(2 \times 34+1)=69$ unknowns for each dataset. Such a high number of unknowns may cause an overinterpretation of the shorter datasets, e.g. for the six nights of data available for the year 1978. The most common effect of the overinterpretation is to spuriously increase the amplitudes for the different modes through noise and aliasing. This problem cannot be treated with the available formal statistical tests, but its effect can be minimized by introducing minimum amplitude limits to be determined experimentally. This Brute Force Method of computing fits with all 34 frequencies should be applied with great caution.

A more conservative approach is provided by the Fourier Peak Method. If $n$ frequencies are already known for a particular dataset, a least-squares algorithm (Breger 1990) fits these $n$ simultaneous frequencies. Then the power spectrum of the residuals is computed from the residuals. The main peaks in this power spectrum are then examined for statistical significance and agreement with known frequency values.

We have applied both methods to the available datasets (Landolt 1966, Danziger \& Dickens 1967, Hayes \& Heiser 1968, Shaw 1977, Fitch 1980, Loumos 
1980, Warman, Peña, \& Arellano Ferro 1979, Breger et al. 1990, 1999, Breger \& Hiesberger 1999). Personal judgement was used to choose only the modes visible in the dataset for the particular year(s). For these modes a multifrequency solution using the program package PERIOD90 was carried out (Breger 1990). Due to their small amplitudes, no combination frequencies, $\mathrm{f}_{i} \pm \mathbf{f}_{j}$, were detected. Fig. 1 shows the results for the other modes covering the years 1966-97.

\section{4 CVn: Phase Shifts and Amplitude Variability of the $7.375 \mathrm{~d}^{-1}$ Mode}

The amplitude and phase changes of the $7.375 \mathrm{~d}^{-1}$ mode of $4 \mathrm{CVn}$ are fascinating (see Figure 2): the amplitude dropped from $15.2 \mathrm{mmag}$ in 1974 to $3.6 \mathrm{mmag}$ during 1976 and $1.4 \mathrm{mmag}$ in 1977 , while it increased again to $7.5 \mathrm{mmag}$ during 1978. The amplitude drop is the most rapid change observed so far in $4 \mathrm{CVn}$. We also find:

(i) The mode is present during all four observing seasons. Even at minimum amplitude during 1977 (1.4 mmag), it shows a definite peak in the power spectrum after the other modes have been prewhitened.

(ii) No single frequency value can represent the four years without a large phase jump or some negative amplitudes (implying a 0.5 cycle phase shift).

(iii) Phasing the 1974 to the 1976 data gives a frequency of $7.3752 \mathrm{~d}^{-1}$. This value is in exact agreement with the value derived from the large 1996/7 dataset, and the value found from the long 1974 dataset alone, which covers $123 \mathrm{~d}$. The 1977 to 1978 data also give $7.3752 \mathrm{~d}^{-1}$, but shifted in phase by $0.48 \pm 0.02$ cycles relative to $1974-1976$.

We conclude that the frequency was essentially constant from 1974-78 with its standard value of $7.3752 \mathrm{~d}^{-1}$. Between 1974 and 1976 the amplitude dropped by a factor of four. A year later, the mode was observed again with an even smaller amplitude and phase-shifted by about half a cycle. The amplitude then increased rapidly to the observed value of $8 \mathrm{mmag}$ in 1978 . This observed behavior cannot be explained by the observational uncertainties, since these are much smaller than the observed changes. Note also that the large gaps ( $\sim$ a decade) in the 1966-97 data prevent the derivation of unique, accurate frequency values from the analysis of the combined data.

The observed phase shift between 1976 and 1977 of almost half a cycle suggests beating of two close frequencies of similar amplitudes. We have modeled this possibility with the least-squares multifrequency fitting program. The Simple Beating Model fails to reproduce the observed behavior.

An alternative explanation is the Re-excitation Model: In this model the $7.375 \mathrm{~d}^{-1}$ mode decayed in amplitude and died out completely between 1976 and 1977 . The mode was re-excited very shortly thereafter, but with a randomly different phase.

We also note that the $7.375 \mathrm{~d}^{-1}$ mode shows the strongest mode coupling of all modes. The amplitudes of these combination frequencies are also variable from year to year. The presence of both strong amplitude variability and strong mode coupling suggests that the amplitude variability is caused by power transfer between modes. 


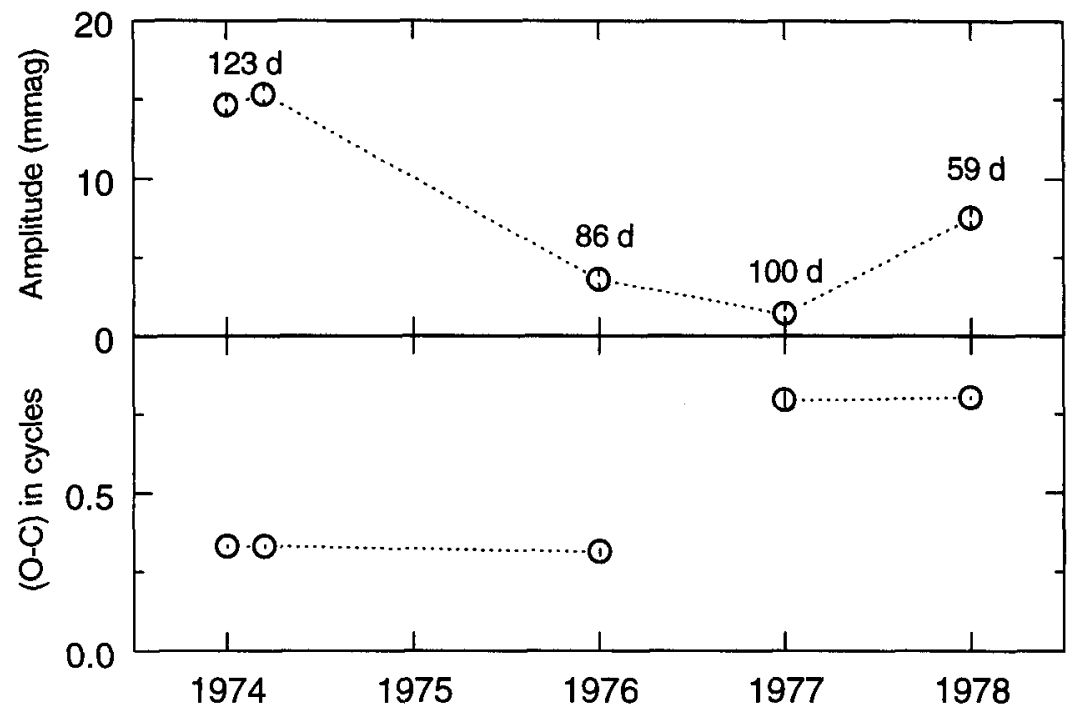

Figure 2. Amplitude variability and phase shifts of the $7.38 \mathrm{~d}^{-1}$ mode using the frequency value of $7.37521 \mathrm{~d}^{-1}$ accurately determined from the 1996-7 data. The formal errors are the vertical lines inside the open circles. The length of the annual measurements are given by the numbers above the circles. The data indicate that a sudden phase shift occurred between 1976 and 1977 when the amplitude decayed.

Acknowledgments. Part of this investigation has been supported by the Austrian Fonds zur Förderung der wissenschaftlichen Forschung, project number S7304.

\section{References}

Breger, M. 1990, Comm. in Asteroseismology (Vienna), 20, 1

Breger, M., Handler, G., Garrido, R., et al. 1999, A\&A, in press

Breger, M. \& Hiesberger, F. 1999, A\&AS, 135, 547

Breger, M., McNamara, B. J., \& Kerschbaum, F. 1990, A\&A, 231, 56

Danziger, I. J. \& Dickens, R. J. 1967, ApJ, 149, L55

Fitch, W. S. 1980, Lecture Notes in Physics, 125, 7

Hayes, J. E. \& Heiser, A. M. 1968, PASP, 80, 57

Landolt, A. U. 1966, PASP, 78, 51

Loumos, G. 1980, Ph. D. Dissertation, Univ. of Texas at Austin

Mantegazza, L. \& Poretti, E. 1996, A\&A, 312, 855

Shaw, J. S. 1977, AJ, 82, 42

Warman, J., Peña, J. H., \& Arrelano Ferro, A. 1979, AJ, 84, 109 


\section{Discussion}

Pawel Moskalik: The phase jump you have found can be also a result of a mode coupling. In my paper of 1985 I have found that such a jump occurs at the same time when the modulated mode goes through the amplitude minimum. This was shown for the 2:1 parametric resonance.

Michel Breger: Your hypothesis on the phase shift of the $7.38 \mathrm{~d}^{-1}$ mode of $4 \mathrm{CVn}$ is very attractive because for this mode the coupling with other modes is very strong and shows high amplitudes of $f_{i}+f_{j}$ and $f_{i}-f_{j}$.

Don Kurtz: I'd just like to emphasize your comment that fitting the light curves is not proof of what the star is really doing. It only proves you have flattened the amplitude spectrum. 